\title{
Pharmacist Mediated Assessment and Reporting of Adverse Drug Reactions in a Tertiary Care Hospital
}

\author{
Inamdar Syed Zia ${ }^{1, *}$, Pradeepthi K, Ashwini N², Soumyashree L², Kulkarni R.V ${ }^{1}$ \\ ${ }^{1}$ Department of Pharmacy Practice, BLDEA's SSM College of Pharmacy and Research Centre, Vijaypur 586 103, Karnataka, INDIA. \\ 2Intern Pharm D, BLDEA's SSM College of Pharmacy and Research Centre, Vijaypur 586 103, Karnataka, INDIA.
}

\begin{abstract}
Background: ADRs are one of the foremost reasons of illness and death, adding to overall preventive medicine cost. Reporting of such adverse drug reactions are critical parameter of medical treatment. The present study assess the clinical pharmacist role in drug monitoring to detect and intercept adverse drug reaction in a health care setting, Method: A prospective observational was conducted at tertiary care hospital in Vijaypur city. A total of 50 suspected adverse effects were recognized and documented during the study period of six months. The suspected adverse effects were assessed for its causality and severity by using Naranjo's and Hartwig's scale. Results: Overall 50 ADRs were identified and documented during the study period. Most of the reported ADRs in this study were Type A 34(68\%). Drug dose and frequency 18(33.96\%), Age 15(28.3\%) and polypharmacy $10(18.86 \%)$ were the furthermost protruding predisposing factors of ADRs were observed. Antibiotics were the common class of drugs involved in producing ADRs $14(28 \%)$. Causality assessment by Naranjo's scale presented that majority of the reported ADRs were found to be probable $26(52 \%)$, possible $19(38 \%)$ and definite $3(6 \%)$. Severity assessment by Hartwig's scale showed that 26(52\%) ADRs were moderate, 17 (34\%) ADRs were mild and $7(14 \%)$ ADRs were of severe. Withdrawal of the drug $27(54 \%)$ as a management intervention was the core line of the adverse drug effect management. Conclusion: Clinical Pharmacist preparedness towards pharmacovigilance approach and proximity for drug monitoring resulted in greater interception and reporting of adverse effect ensuring patient drug related safety.
\end{abstract}

Key words: Adverse drug reaction, Pharmacovigilance, Severity, Causality, Clinical pharmacist.

\section{INTRODUCTION}

The concept of ADR (Adverse Drug Reaction) monitoring and reporting system in India is out there for a considerable time and slowly but steadily gaining its due importance in drug safety measures. Deficiency in real ADR reporting and monitoring in Indian population for drug safety is still a huge challenge among health care professional. Therefore, to increase the reporting rate, it is important to improve the knowledge, attitude and practice of healthcare professionals regarding ADR reporting and pharmacovigilance. ${ }^{1} \mathrm{WHO}$ defines the Pharmacovigilance (PV) as "the pharmacological science relating to the detection, evaluation, understanding and prevention of adverse effects, particularly long term and short-term side effects of medicine." Every country has established their individual set of guidelines on Pharmacovigilance for detection, collection, assessment of adverse events in their corresponding regions. The final aim of Pharmacovigilance is to safeguard safe and rational use of medications. "No drug absolutely is free from side effects" which is universally accepted statement. ${ }^{3}$ According to World Health Organization (WHO), the term ADR can be defined as "Any response to a drug which is noxious and unintended, and which occurs at doses normally used in man for prophylaxis, diagnosis, or therapy of disease or for the modification of physiological function". Pharmacovigilance plays a significant part in the investigation of adverse drug reactions, a significant reason of illness and death. Present epidemiological studies assessed that ADRs are fourth to
DOI: 10.5530/ijopp.11.3.28

Address for correspondence: Dr. Syed Zia Inamdar, Associate Prof and Clinical Pharmacist, Department of Pharmacy Practice, BLDEA's SSM College of Pharmacy and Research Centre, Vijaypur 586 103, Karnataka, INDIA.

Phone no: 9986666016 Email Id: syedzia.inamdar@ gmail.com

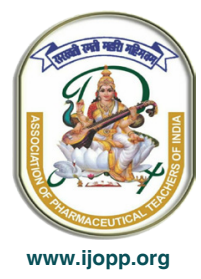


sixth foremost root of death. ${ }^{4-5}$ ADRs causes important economic burden on national health budget. It raises costs of patient care and may mimic disease leading to unnecessary investigations and delay in treatment. ${ }^{5}$ So there is a need to study ADRs seriously, to create consciousness about it among patients, to encourage healthcare professionals in reporting ADRs to reduce the risk. ${ }^{6}$ The pharmacist and the prescriber have a duty to safeguard patients with the risk of untoward drug effects. The pharmacist are appropriately placed in the whole medication use process, are accessible and important link between the patient and the physician. A thorough knowledge of medicine and drug monitoring skills of pharmacist plays major role in the promotion of drug safety and optimizing patient outcomes. ${ }^{1}$ The present study aims to recognize and illustrate the pattern of ADRs due to frequently used drugs with their possible contributing factors from the prospective role of a clinical pharmacist in drug monitoring and ADRs management.

\section{MATERIALS AND METHODS}

A prospective observational study was carried out for a period of six months in the selected departments of a tertiary care hospital in Vijaypur city. The study was approved by Institutional Ethical Committee. The inpatients of the selected department who are receiving treatment in the hospital at the time of the study were routinely monitored by the clinical pharmacist on day to day basis. Any episode of suspected ADRs detection or its occurrence was reported and documented by the pharmacist on a standard data collection form prepared in accordance to CDSCO ADR reporting template. Supplementary information was also extracted by interacting with the patient's and reviewing patient case files (including patient's specific information, laboratory investigations and drug therapy). The suspected ADR was further followed for its management and assessed for its causality and severity using Naranjo's and Hartwig's scale.

\section{RESULTS}

Overall 50 ADRs were identified and documented during the six months study period. Among them $4(8 \%)$ inpatients were admitted due to ADR reason and during their hospital stay $46(92 \%)$ patients developed ADR. Among the reported ADRs in terms of patient demographics, $30(60 \%)$ were male and $20(40 \%)$ were female. The majority of the ADRs were of Type A $34(68 \%)$ followed by Type B 14(28\%) and Type F 2 (4\%) [Figure 1]. Of the reported ADRs, the prominent predisposing factor was drug dose and frequency $18(33.96 \%)$ followed by age 15(28.3\%), polypharmacy
10(18.86\%), therapeutic index of drug 4(7.54\%), allergy 4(7.54\%), smoking $1(1.88 \%)$ and Alcohol $1(1.88 \%)$ [Figure 2]. The most common therapeutic class involved in ADRs was Antibiotics 14(28\%) while others were steroids $5(10 \%)$, antihypertensive drugs $4(8 \%)$, NSAIDS $3(6 \%)$, anticoagulants $3(6 \%)$, antiviral $3(6 \%)$, antiulcer drugs $2(4 \%)$, anti-malarial2 $(4 \%)$, antitubercular $2(4 \%)$, diuretics $2(4 \%)$, neurotransmitters $2(4 \%)$, bronchodilators $2(4 \%)$, antiepileptic $1(2 \%)$, antifungal $1(2 \%)$, anti-Hyperlipidemia $1(2 \%)$, mucolytics $1(2 \%)$, antihistamines $1(2 \%)$ and vitamin supplement $1(2 \%)$ [Figure 3]. Causality assessment by Naranjo's scale showed that out of 50 ADRs, $26(52 \%)$ were probable, $19(38 \%)$ were possible, $3(6 \%)$ were definite and 2(4\%) were doubtful. [Figure 4] By using Hartwig's scale, Severities of the reactions were assessed. Among the reported ADRs, $26(52 \%)$ were moderate reactions followed by $17(34 \%)$ were mild reactions and $7(14 \%)$ were severe [Figure 5]. Management of ADRs in the study showed that out of 50 ADRs, 27(54\%) ADRs were managed by withdrawing suspected drug, 13(26\%) ADRs were managed by adding a supplement, 12(24\%) ADRs were managed by replacing a drug, 2(4\%) ADRs were managed by altering the dose while no change was made in 2(4\%) ADRs [Figure 6].

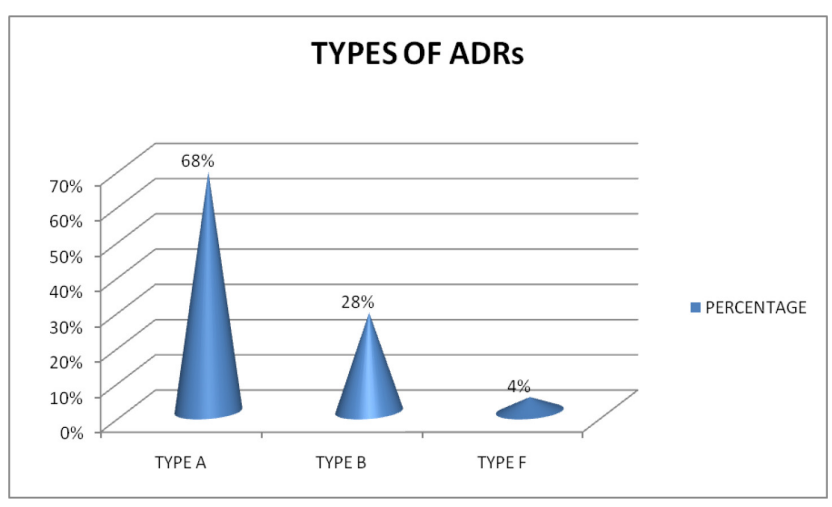

Figure 1: Types of Adverse Drug Reactions.

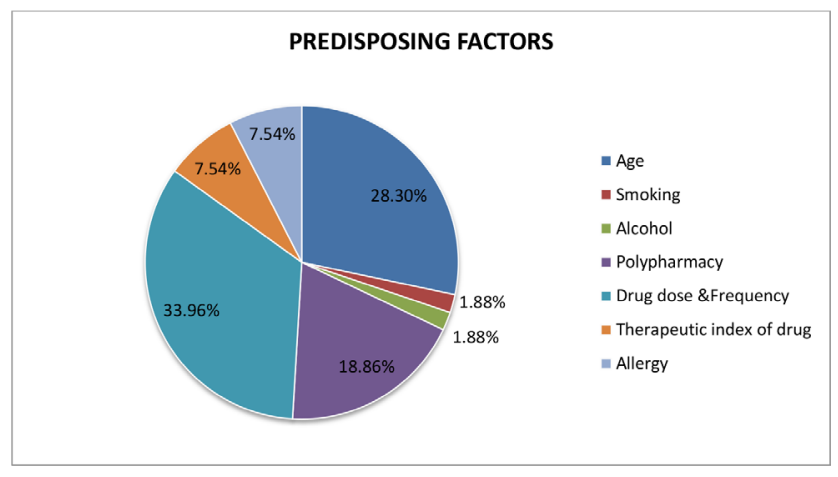

Figure 2: Predisposing factors of Adverse Drug Reactions. 


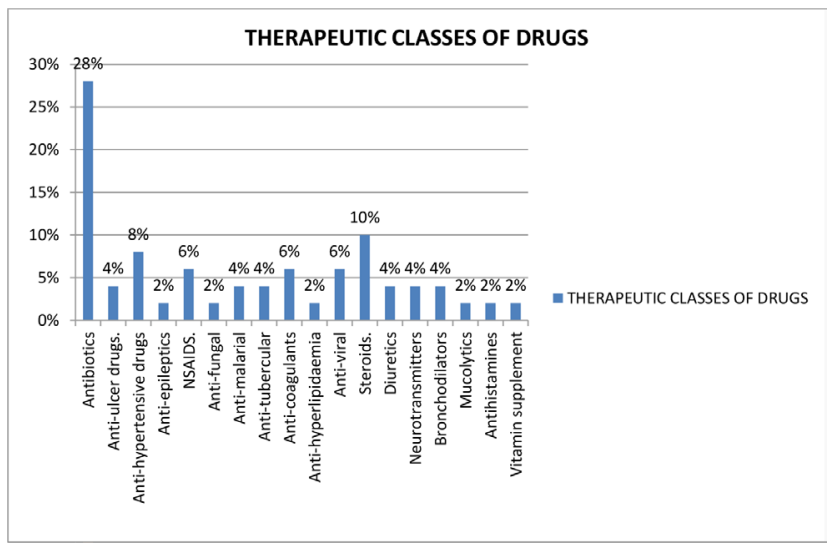

Figure 3: Therapeutic classes of drug wise distribution of Adverse Drug Reactions.

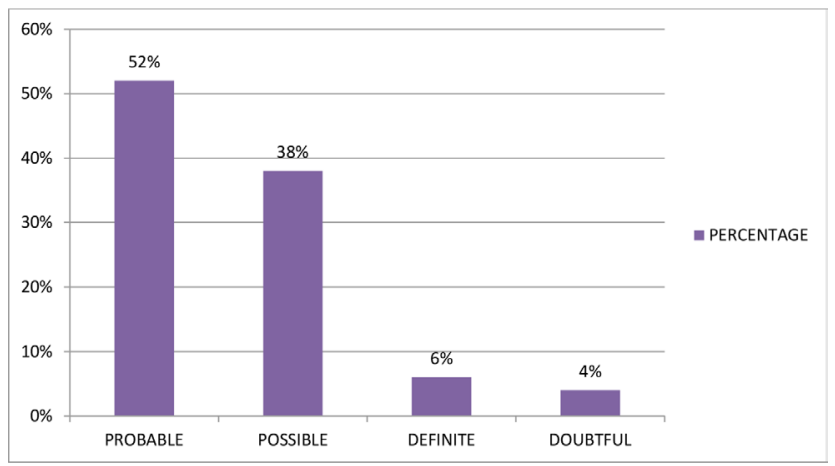

Figure 4: Causality assessment of Adverse Drug Reactions (Using Naranjo's scale).

\section{DISCUSSION}

A total of 50 suspected ADRs were identified and documented during this six months study period. It was found out that there were more number of ADRs in males $30(60 \%)$ compared to females $20(40 \%)$ in our hospital. This may be due to majority of in-patients were male with more antibiotic use during the study period. Some of the factors like smoking and alcohol intake may also be an important risk factor to develop ADRs more in male gender. This result is reliable with the results of the study carried out by Shamna M et al. ${ }^{7}$ The analysis of the types of reported ADRs revealed that more number of ADRs were of type A comprising about 34(68\%) followed by type B comprising about $14(28 \%)$ and followed by type F of 2(4\%). Drug dosing and frequency plays a vital role in the development of ADRs as type A reactions are dose related and therefore, can be preventable from their known pharmacology and type B reactions mostly include hypersensitivity reactions. Even though, majority of the reactions we found are of moderate type, due to this reason the health care cost were increased which was due to an increased length of stay and necessity of some medical interventions as a result of prevalence of adverse drug reactions. This result was reliable with the

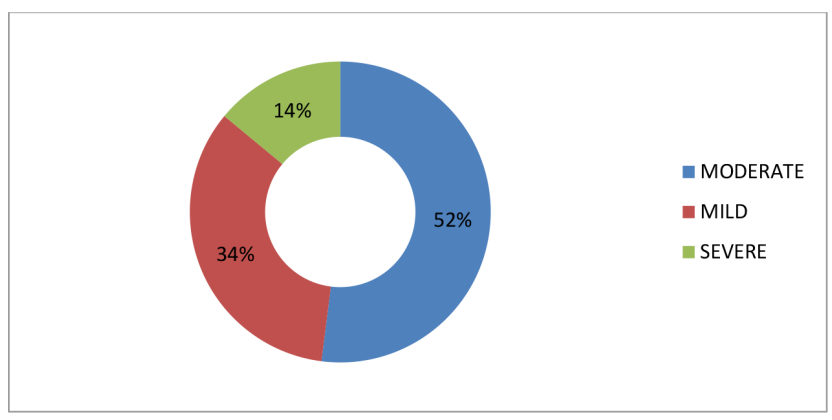

Figure 5: Level of severity of reported Adverse Drug Reactions (using Hartwig's Scale).

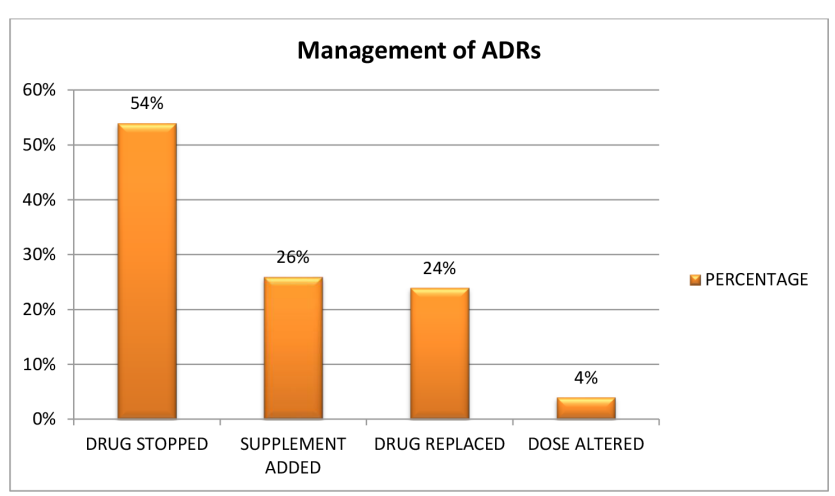

Figure 6: Management of Adverse Drug Reactions.

study carried out by Shamna M et al. ${ }^{7}$ Drug dose and frequency $18(33.96 \%)$, age $15(28.3 \%)$, polypharmacy $10(18.86 \%)$ were the utmost protruding predisposing factors of ADRs. A total of 50 suspected ADRs were identified during study period and predisposing factors tend to be 53 , this may conclude that an ADR occurring in some individual patients may be due to exposure of more than one predisposing factor. There might be some effect on the development of ADRs thus, dosing needs should be considered as a factor. Associated patient's disease may also effect susceptibility to ADR's as majority of the patients who developed ADRs were having co-morbidities like diabetes, hypertension, COPD, bronchial asthma, cirrhosis, tuberculosis etc., necessitating them to receive multiple drugs, which contributes to the increased risk of ADRs. In order to prevent potential ADRs which affect patient health status, compliance and therapeutic outcomes polypharmacy should be considered. This result was inconsistent to a study conducted by Dilip C et al. ${ }^{4}$ This study revealed that antibiotics were the most commonly implicated drug class as antibiotics drugs are highly consumed in our hospital to treat various diseases, which is similar to a study conducted by Shamna M et al. ${ }^{7}$ Most of the antibiotics are known to 
cause GI related side effects and GIT was found to be the most affected organ in our study for the precipitation of ADRs which ultimately lead to GI side effects (loose motions, vomiting and abdominal pain). Causality was assessed as per Naranjo's Scale through patient interview and interaction with doctors and healthcare professionals. According to Naranjo's scale, probable were 26(52\%), possible $19(38 \%)$, definite $3(6 \%)$ and doubtful $2(4 \%)$ which is similar to a study conducted by Khan A et al..$^{8}$ The assessment and establishment of causality relationship between suspected drugs and reactions can help in signal generation and drug regulation. This serves as useful reference to alert clinicians to the likelihood of a particular drug causing a suspected reaction. To assess the level of severity of reported ADRs Hartwig's scale was used, which revealed that moderate cases were $26(52 \%)$, mild cases were $17(34 \%)$ and $7(14 \%)$ of severe cases, which is similar to a study conducted by Padmavathi $\mathrm{S}$ et al. ${ }^{9}$ Most of the patients on multiple drug therapy developed more number of moderate level severity reaction than others. Drug stopped 27(54\%) was the foremost line of management of ADRs while supplement added were $13(26 \%)$, drug replaced $12(24 \%)$, dose altered $2(4 \%)$ and no change was made with the suspected drug in $2(4 \%)$. This result was reliable with the study carried out by Patidar $\mathrm{D}$ et al..$^{10}$

\section{CONCLUSION}

The lively participation of clinical pharmacists can plays a significant role in the identification and documentation of ADRs by virtue of their role in patient drug monitoring. The health care prescriber's has diminutive ADR reporting culture due to patient overload and to a certain extent a deficiency of interest to report ADRs. ADRs are unique drug related problems in the hospital setting and preventing them is a task for safeguarding drug safety. Major number of ADRs was caused by antibiotic drugs due to advanced prescription use of antibiotics for prophylaxis and treatment of various diseases. The healthcare system should encourage and adopt clinical pharmacy services rendered by clinical pharmacist to promote drug safety and rational use of drugs among patient population to optimize treatment outcomes.

\section{ACKNOWLEDGEMENT}

The authors are thankful to the management of BLDE association for supporting the work.

\section{CONFLICT OF INTEREST}

The authors declare no conflict of interest.

\section{ABBREVIATIONS}

ADR: Adverse Drug Reactions, WHO: World Health Organization, PV: Pharmacovigilance, CDSCO: Central Drug Standard Control Organization, NSAID: Non Steroidal Anti- Inflammatory Drugs, GI: Gastro Intestinal, COPD: Chronic Obstructive Pulmonary Disease, GIT: Gastro Intestinal Tract.

\section{SUMMARY}

Drug safety measures are still a huge challenge and contribute significantly to the health and economic burden in a patient care process. The deficient reporting culture of ADRs by health professionals further potentiates the problem. The pharmacist, through its apparent role in drug monitoring can assist and promote drug safety and safeguard patients with the risk of untoward drug effects.

\section{REFERENCES}

1. Lihite RJ, Lahkar M. An update on the Pharmacovigilance Programme of India. Front Pharmacol. 2015;6(194). doi: 10.3389/fphar.2015.00194

2. Rabbur RSM, Emmerton L. An introduction to adverse drug reaction reporting system in different countries. Int J Pharm Prac. 2005;13(1):91-100.

3. Sulthan MS, Bawazir SA. Adverse drug reporting by Hospital Pharmacist in Saudi Arabia. Saudi Pharmaceutical J. 2009;17(1):95-105.

4. Dilip C, Lisa MM, Saraswathi R, Divya R. Adverse drug reaction monitoring in a tertiary level referral hospital, Kerala. Ind J Pharm Prac. 2012;5(2):28-32.

5. Srinivasan R, Ramya G. Adverse Drug Reaction-Causality Assessment. Int J Res Ph and Chem. 2011;1(3):606-12.

6. American Society of Health-System Pharmacists. ASHP guidelines on Adverse drug reaction monitoring and Reporting. An J Health-Syst Pharm. 1995;52(4):417-9

7. Shamna $\mathrm{M}$ et al. A prospective study on adverse drug reactions of antibiotics in a tertiary care hospital. Saudi Pharmaceutical Journal. 2014;22(4):303-8. doi: 10.1016/j.jsps.2013.06.004

8. Khan A, Mir SA, Nematullah K, Ihtisham S, Aamer K, Aamir S. Causality assessment of adverse drug reaction in Pulmonology Department of a Tertiary Care Hospital. Journal of Basic and Clinical Pharmacy. 2015;6(3):84-8. doi: 10.4103/0976-0105.160744

9. Padmavathi S, Manimekalai K, Ambujam S. Causality, Severity and Preventability assessment of adverse cutaneous drug reaction: A prospective observational study in a Tertiary Care Hospital. J Clin Diagn Res. 2013;7(12):2765-7. doi: 10.7860/JCDR/2013/7430.3753

10. Patidar D, Rajput MS, Nirmal NP, Savitri W. Implementation and evaluation of adverse drug reaction monitoring system in a Tertiary Care Teaching Hospital in Mumbai, India. Interdiscip Toxicol. 2013;6(1):41-6. doi: 10.2478/ intox-2013-0008

11. Gupta SK, Nayak RP, Shivaranjani R, Vidyarthi SK. A questionnaire study on the Knowledge, Attitude, and Practices of Pharmacovigilance among health care professionals in a teaching hospital in South India. Perspect Clin Res. 2015;6:45-52. 